\title{
Research on evaluation method of energy supply reliability of regional energy Internet
}

\author{
Li Pengli ${ }^{1, *}$, Su Jian ${ }^{1}$, Yan Tao $^{1}$, Liu Jiqiu ${ }^{2}$, and Qi Xiaoguang ${ }^{3}$ \\ ${ }^{1}$ China Electric Power Research Institute, Haidian District, Beijing, China \\ ${ }^{2}$ State Grid Hebei Electric Power Company, Shijiazhuang, Hebei Province, China \\ ${ }^{3}$ State Grid Hebei Economic Research Institute, Shijiazhuang, Hebei Province, China
}

\begin{abstract}
This paper studies the reliability evaluation technology of regional energy Internet and represents a reliability evaluation method considering the optimal load reduction strategy. The application scenario of regional Energy Internet with power system as the core is built. The specific form is that multiple Integrated Energy systems are connected to the superior distribution network through the tie line. The reliability evaluation index system of power system and regional energy Internet is introduced. Based on the modelling of components in the regional energy Internet application scenario in this paper, the operation model and two-state Markov model are mainly established for the renewable energy power generation devices, energy coupling devices and energy storage devices in IES. The optimal load reduction models based on load classification are established for the faults of distribution network lines and the faults of IES internal components connected to the distribution network in regional energy Internet respectively. Through the construction of different application scenarios, the load reduction situation under different faults and different component parameters is analysed, and the corresponding reliability index calculation and result analysis are carried out.
\end{abstract}

\section{Introduction}

Since the second industrial revolution, the rapid economic development brought by electrification has led to the environmental damage crisis and energy shortage worldwide. To solve the energy crisis, most countries in the world have reached a consensus. On the one hand, the development and utilization technology of renewable energy should be developed as soon as possible to promote the penetration of various forms of new energy in the energy network. On the other hand, for the traditional single energy management system such as electric power, gas and heat, the comprehensive coordinated planning should be promoted to build a multi-energy network coupling system with power network as the core to improve the overall energy efficiency of the system [1]. It can realize central and distributed coordination of energy structure, horizontal optimization and coordination of various types of energy forms, vertical coordination of energy supply and consumption, and advocate a new ecological energy system with extensive user participation.

Research on the reliability of regional energy Internet has started, and some research results have been published. Ref. [2] presented the equipment state transfer model based on Markov process. Ref. [3] established the Markov two-state model of photovoltaic and CHP equipment in the integrated energy micro grid to describe the normal and fault states of the unit equipment. Ref. [4] studied the Markov stochastic state transfer model of fan, photovoltaic, CHP, energy storage and other equipment in the integrated energy system, so as to facilitate the evaluation of system reliability by using the state-space transfer method. Ref. [5] modelled the fault load reduction of the electric-pneumatic coupling system considering the energy storage operation strategy. Ref. [6] established a two-stage optimization model to simulate the operation strategy of industrial automation system and evaluate the energy supply capacity under the scenario of random generation. Ref. [7] adopted sequential Monte Carlo method to simulate gas-power coupling network, and calculates reliability indicators such as load loss probability and expected power supply shortage of gas-power combined network by minimizing the comprehensive cost of natural gas and power network. On the above research, Ref. [8] used Monte Carlo method of Markov process to evaluate the reliability of integrated energy system.

Aiming at the regional energy Internet architecture with power system as the core, this paper proposes a reliability evaluation method considering the optimal load reduction strategy, which is used to calculate the reliability of the multi-energy coupled energy Internet with distribution network as the core. 


\section{Materials and Methods}

\subsection{The framework of regional energy Internet}

Regional energy Internet is mainly composed of energy generation module, energy exchange module, energy storage module and a large number of user energy consumption units. This paper constructs an energy Internet form in which IES is connected with regional energy network through different tie lines. This system architecture is shown in Fig.1 Later focuses on the situation of the distribution of multiple access IES, as shown in Fig. 2 regardless of the natural gas and thermal network internal connecting method, the boundary of the power distribution network and the connection in different location in distribution network of multiple IES, IES contains links to external gas network, heat supply network of energy coupling device and the corresponding electricity, gas, heat/cooling load, Multienergy coupling and conversion are realized in IES, and the reliability evaluation of this energy Internet architecture is studied in this paper.

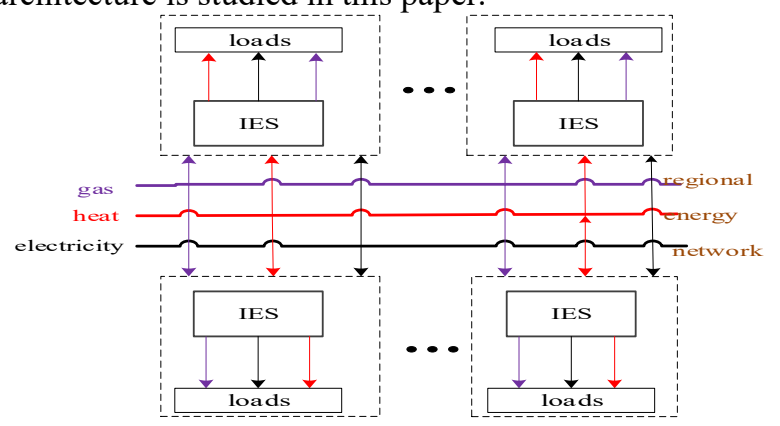

Fig. 1. Multiple IES are connected in the regional energy network

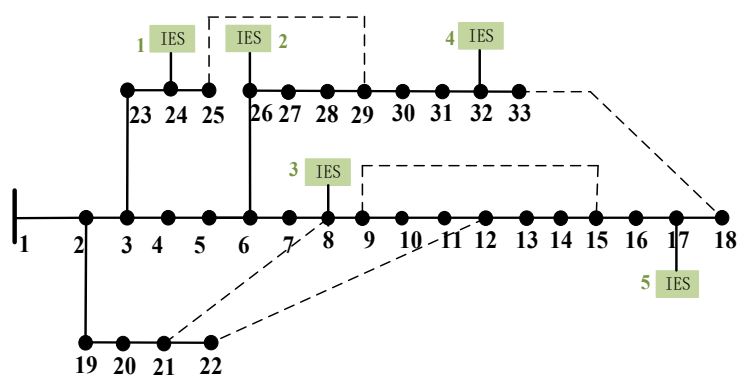

Fig. 2. Regional energy Internet with distribution network as the core

\subsection{Two-state Markov Monte model}

Two states of normal and failure of circuits in distribution network and various energy coupling devices in IES are considered. The above components are all repairable components, which can return to the normal operation state through the component repair process after failure. Each component is assumed to enter a repair state immediately after a failure, so the flow of state is an alternating of normal and failure states during the lifetime of such a component. The state transition model of two-state components is shown in Fig.3[9]. Within a certain simulation period, the state transition sequence of each component can be obtained by extracting random numbers through Markov Monte Carlo, and then the state of the whole system can be determined.

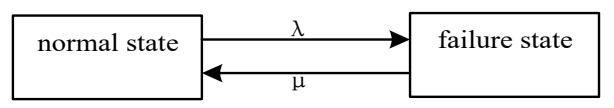

Fig. 3. Two-state element state transition model diagram

In Fig. $3 \lambda$ is the failure rate and $\mu$ is the repair rate. The time to failure (TTF) and the time to repair (TTR) of components follow the exponential distribution with respect to $\lambda$ and $\mu$ respectively. TTF and TTR can be obtained by extracting random numbers between $[0,1]$, as shown in Eq. (1).

$$
\left\{\begin{array}{l}
\text { TTF }=-\frac{1}{\lambda} \ln \alpha \\
T T R=-\frac{1}{\mu} \ln \beta
\end{array}\right.
$$

Where $\mathrm{A}$ and $\mathrm{B}$ are random numbers within the value range of $[0,1]$ and subject to uniform distribution.

\subsection{Fault optimal load reduction model}

The optimal load reduction models of these two faults are studied respectively.

\subsubsection{Failure analysis of main network frame of distribution network in regional energy Internet}

After the failure of distribution network in regional energy Internet, the fault recovery process of the system can be generally divided into two stages: distribution network fault reconstruction and IES support distribution network. Therefore, the optimal load reduction model of distribution network fault is presented.

The optimization objective of the reconfiguration of regional energy Internet distribution network frame is to minimize the weighted value of the total power loss load of the distribution network, minimize the IES lost connection with the main network, minimize the network loss, and minimize the number of switching operations. The optimization variable is the switching state in the distribution network, and its objective function is:

$$
F=\min \left(\omega_{1} f_{1}^{\prime}+\omega_{2} f_{2}^{\prime}+\omega_{3} f_{3}^{\prime}+\omega_{4} f_{4}^{\prime}\right)
$$

In the Eq.(2), $f_{1}^{\prime}, f_{2}^{\prime}, f_{3}^{\prime}$ and $f_{4}^{\prime}$ are respectively the normalized values of $f_{1}, f_{2}, f_{3}$ and $f_{4}$ (converting to the interval $[0,1])$, in order to eliminate the influence of different value units of each objective function on the optimization results.

$$
\begin{gathered}
f_{1}=\min \sum_{k=1}^{N} \alpha_{k} P_{k} \\
f_{2}=\min \sum_{i=1}^{M} P_{i}^{I E S}
\end{gathered}
$$




$$
\begin{gathered}
f_{3}=\min \sum_{i, j=1}^{B} R_{i j} \frac{P_{i j}^{2}+Q_{i j}^{2}}{U_{j}^{2}} \\
f_{4}=\min \left(\sum_{i=1}^{D}\left|r_{i}-b_{i}\right|\right)
\end{gathered}
$$

Where, $f_{1}, f_{2}, f_{3}$ and $f_{4}$ are the load-weighted value function of power loss network nodes, the number function of IES lost in the main network, the network loss function, and the switching frequency function respectively. $P_{k}$ is the power value of load $k ; \alpha_{k}$ is the weight of the importance degree of the load, and the load is divided into important load, relatively important load and general load according to the importance degree. $N$ is the collection of power loss load; $M$ is a collection of nodes of IES that have lost connection to the primary network; $B$ is the node set of distribution network; $D$ is the set of distribution network switches; $r_{i}$ and $b_{i}$ are respectively the states before and after the failure recovery of the first switch, which is the variable 0 or 1 , 0 represents the switch off, 1 represents the switch closed.

In order to realize the supporting role of IES in the fault recovery process of regional energy network, an internal optimal scheduling model of IES was established with the interactive power of the connecting pipeline between IES and regional energy network as the decision variable. Under the premise of reducing the amount of load as far as possible, considering the output constraint of energy supply equipment, meeting the power transmission requirements with regional energy network, reducing the input power of the connecting pipeline from regional energy network, achieving the goal of giving consideration to both economy and energy supply reliability. The objective function is like Eq. (7).

$$
F_{x}^{k}=\min \sum_{i=1}^{n}\left(\omega_{i} \Delta P_{i}^{x}\right)
$$

Where, $n$ is IES number; Operating state $x$ corresponds to the operation scheduling stages of IES loads with different importance degrees (interruptible load, general load, relatively important load, and important load); $\Delta P_{i}^{x}$ is the actual adjustment amount of the tie line power of the first IES under the condition of operating state $x ; \omega_{i}$ is the weight coefficient.

At the same time, the grid node in each phase of the power cut allocation strategy is: the first is the failure outage regional grid node load, is insufficient to consider in a distribution network fault power network reconstruction after connecting the area on the connecting line of the grid node load, is still insufficient to consider other position of the node load of power grid. The allocation objective function is like Eq. (8).

$$
F_{y}^{k}=\min \sum_{i=1}^{n}\left(\omega_{i}^{y} P_{i}^{y}\right)
$$

Where, $i$ is the grid node number; $P_{i}^{y}$ is the load value under the running state y of node $i$; Operating state y corresponds to the operation scheduling stages of loads of different importance degrees (third-level load, secondlevel load and first-level load); $\omega_{i}^{y}$ is the weight coefficient.

\subsubsection{Analysis of internal failures of regional energy Internet IES}

When a device in the integrated energy system fails, the output power of the component immediately becomes zero, and the corresponding output energy is zero. In order to minimize both load reduction and system operating cost, the objective function of this paper is to minimize the sum of load reduction cost and operating cost.

$$
\left\{\begin{array}{l}
\min F=\left[C_{c u t}(t)+C_{o p}(t)\right] \cdot \Delta t \\
C_{c u t}(t)=K_{\text {ecut }} c_{\text {elec }} L_{\text {ecut }}(t)+K_{\text {hcut }} c_{\text {heat }} L_{\text {hcut }}(t) \\
C_{\text {op }}(t)=C_{\text {ele }}(t)+C_{\text {gas }}(t)
\end{array}\right.
$$

Where, $\Delta t$ is the operation time of the integrated energy system; $C_{c u t}(t)$ and $C_{o p}(t)$ are the load reduction cost and operation cost under the condition of IES failure in time period respectively; $C_{\text {elec }}$ and $C_{g a s}$ are respectively the cost of IES purchasing and selling electricity from the power grid and the cost of gas online shopping from the gas industry in unit time; $L_{\text {ecut }}$ and $L_{h c u t}$ are respectively the reduction amount of electricity and heat load during the period; $c_{\text {elec }}$ and $c_{\text {gas }}$ are respectively electric energy (purchase and sale price) and gas energy price; $K_{\text {ecut }}$ and $K_{h c u t}$ are respectively the cost coefficient of electric and thermal load reduction, and the value is large to ensure that the integrated energy system will not carry out such load reduction for the purpose of economy in the case of failure, leading to the reduction of the reliability of the energy supply of this kind of energy.

$$
\begin{gathered}
C_{\text {ele }}(t)=c_{\text {ele }} \cdot P_{\text {ele }}(t) \\
C_{\text {gas }}(t)=c_{\text {gas }} \cdot\left[\frac{P_{M T}(t)}{\eta_{M T} L_{H V N G}}+\frac{Q_{G B}(t)}{\eta_{G B} L_{H V N G}}\right]
\end{gathered}
$$

The input terminal of the integrated energy system is connected with the power grid and natural gas network. The input electric quantity and natural gas volume are subject to the maximum power transmission limit of the connection line in unit time, and the constraints are expressed as follows:

$$
\left\{\begin{array}{l}
P_{- \text {elec } \max } \leq P_{\text {elec }}(t) \leq P_{\text {elec } \max } \\
0 \leq F_{\text {gas }}(t) \leq F_{\text {gas max }}
\end{array}\right.
$$

Where, $P_{\text {-elec } \max }$ and $P_{\text {elec } \max }$ are respectively the maximum power transmitted to and absorbed from the power grid by the integrated energy system; $F_{\text {gas }}(t)$ and $F_{\text {gasmax }}$ are respectively the amount of natural gas 
absorbed by the comprehensive energy system into the gas network and the maximum amount of natural gas absorbed by the comprehensive energy system.

The load reduction model is a mixed integer linear programming problem, which can be solved by building the Yalmip platform under the Matlab environment.

\section{Results \& Discussion}

The energy Internet example set up in Fig.2 is used to verify the proposed evaluation algorithm, in which 5 IES are connected to nodes $8,17,24,26$ and 32 of the distribution network through the contact line. The internal structure of IES is shown in Fig.4. The dotted line in the figure represents the contact switch. The initial state of the contact switch is disconnected, and it can be closed in accordance with the corresponding strategy for fault recovery in the stage of distribution network reconstruction. The IES input terminal is connected to the natural gas network and the distribution network. In this model, the internal connection relationship of the natural gas network is not considered, and the connection to the distribution network is the improved IEEE33 node network as shown in Fig.2. The operating parameters and state parameters of the main equipment in the example are shown in Table 1. Sequential Monte Carlo simulation method was used to evaluate the reliability of the above examples, and the simulation time was $1000 \mathrm{a}$.

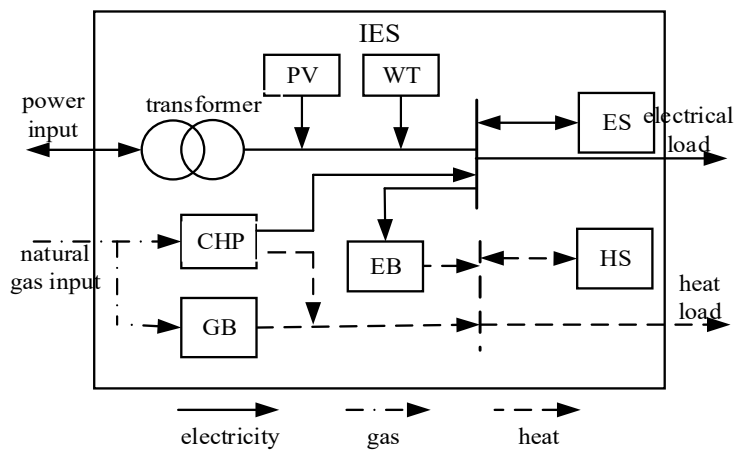

Fig.4. Integrated energy system structure

Table 1. Failure state parameters of main components

\begin{tabular}{|c|c|c|}
\hline Element & Failure rate (/a) & $\begin{array}{c}\text { Mean Time to } \\
\text { repair(h) }\end{array}$ \\
\hline Line & $0.065 / \mathrm{a} \cdot \mathrm{km}$ & 5 \\
\hline Natural gas terminal & 0.025 & 10 \\
\hline Transformer & 0.045 & 5 \\
\hline CHP & 0.02 & 20 \\
\hline Gas storage device & 0.03 & 20 \\
\hline Storage device & 0.02 & 20 \\
\hline Photovoltaic device & 0.03 & 10 \\
\hline Wind generator & 0.03 & 10 \\
\hline Gas boiler & 0.03 & 15 \\
\hline Electric boiler & 0.025 & 15 \\
\hline
\end{tabular}

In order to verify the effectiveness of the IES internal fault load reduction strategy described in Section 2.3, different application scenarios are designed. In order to verify the effectiveness of the IES internal fault load reduction strategy described in Section 2.3, different application scenarios are designed for IES in this section. The power system and gas system are considered to operate separately as scenario 1 . In this scenario, there is no CHP device in IES, the power load is supplied by the electric energy from the generating device through the transformer, and the thermal load is supplied by the gas boiler. In this case, the reduction of electric and thermal load in the system is taken as the basic comparison scenario. The scenario in which the electric and gas systems in IES are coupled but the optimal load reduction strategy is not adopted is considered as scenario 2. In this scenario, the electric and thermal systems are coupled to each other through the CHP unit, but the optimal scheduling strategy is not adopted after the failure of components, and the output of each device is not adjusted. Considering the coupling operation of electricity and gas system in IES and the optimal load reduction strategy in Section 2.3 of this paper, the scenario is taken as scenario 3 . In this scenario, the electric and thermal systems are coupled with each other through CHP unit, and the optimal scheduling strategy is adopted after the failure of components, and the output of each device is optimized for scheduling. In the above scenarios, transformer and gas-fired boiler faults were considered at the same time respectively, and corresponding electrical and thermal load reductions were compared. The comparison results are shown in Fig.5.

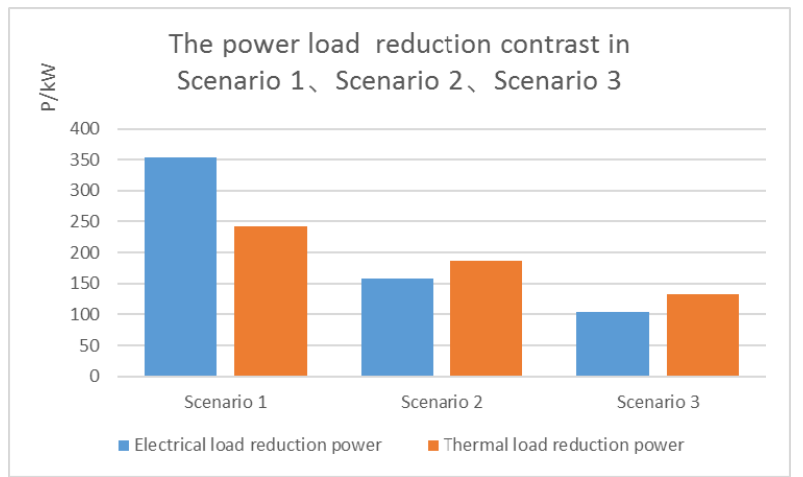

Fig.5. The load reduction contrast in Scenario 1、Scenario 2 and Scenario 3

As can be seen from the analysis in Fig.5, when the electric and gas subsystems operate separately, when the key components fail, the corresponding load supply will be interrupted immediately. Under such a scenario, the power of electric and heat loads will be greatly reduced. When considering the system coupling operation scenario, due to the existence of CHP unit, when the transformer or gas-fired boiler fails, part of the electrical and thermal load in IES can be provided by CHP unit, an electrical, gas-thermal coupling element. Even if the optimal scheduling strategy is not adopted, the power reduction of electrical and thermal loads will be greatly reduced compared to the operating scenario alone. The optimal load reduction strategy is adopted in the coupling system, which can schedule the output of each component and give full play to the multi-energy 
coupling effect of IES under the premise of satisfying the system economy. As can be seen from Fig.5, the optimal load reduction strategy mentioned above can significantly reduce the power reduction in the coupling scenario, thus improving the reliability of power supply and heating of the whole system.

From the above analysis, it can be seen that CHP unit is a key component of IES in regional energy Internet, and the total output of CHP unit is optimized in the scheduling strategy in Section 2.3, so this section analyzes the influence of heat to power ratio on load reduction power. For a specific application scenario at the same time, electricity and heat load with different coefficient of cutting costs, you can also to cut power under certain cost coefficient weighted, to seek the optimal thermoelectric than, electricity, heat load coefficient of cost cutting weight was 0.4 and 0.6 , respectively, with different thermal power than the CHP unit after failure of electricity, heat load power cuts as shown in Fig.6, The total weighted load reduction power under different thermoelectric ratios is shown in Fig. 7.

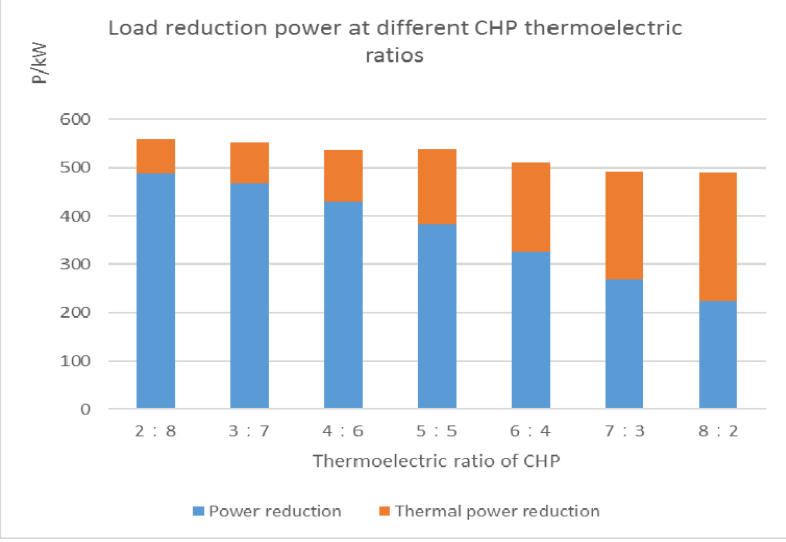

Fig.6. Load reduction of CHP unit failure load under different thermoelectric ratios

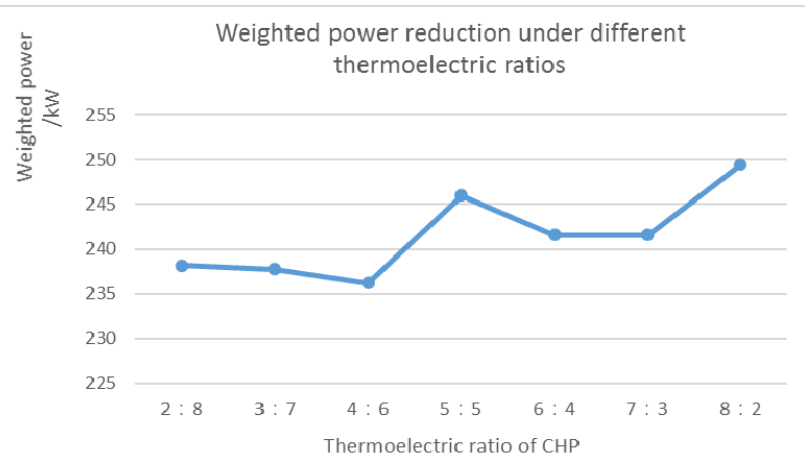

Fig.7. Weighted load reduction under different thermoelectric ratios

As can be seen from Fig. 6, the power reduction of electrical and thermal loads of CHP unit after failure varies in a certain trend under different thermoelectric ratios. For electric loads, the larger the thermoelectric ratio, the smaller the electric power reduction of load; while for thermal loads, the larger the thermoelectric ratio, the greater the thermal power reduction of load. This is because the CHP unit has both power generation and heat generation functions. Considering the objective of fuel gas economy input in the optimal load reduction model, even if the total output of the optimized CHP unit is larger, the power generation of the system is relatively smaller with the greater the thermoelectric ratio, and the greater the power load reduction will be. As can be seen from the Fig.7, by setting the electricity, heat load after cutting costs coefficient of weight, IES fault weighted load cutting power cut first, then increase then decrease again rising trend, the overall when thermoelectric ratio of $4: 6$, the weighted minimum load cutting power, get the load reduction results on optimal reliability and economy, Therefore, the optimum thermoelectric ratio of CHP unit is 4:6.

After the failure of the distribution network feeder, the optimal load reduction strategy in Section 2.3 firstly carries out network reconstruction, and on this basis carries out the joint operation scheduling of distribution network and IES, that is, in this stage, the support of IES for the distribution network failure is considered. In Scenario 4, only the network reconstruction process is considered after the distribution network failure. In Scenario 5, the load reduction scheme proposed in this paper is applied, and IES supports the distribution network while considering the economy and reliability of IES operation. System reliability indexes under the two scenarios are shown in Table 2 and Table 3.

Table 2. System electricity reliability indexes in Scenario 1 and Scenario 2

\begin{tabular}{|c|c|c|c|}
\hline Scenario & EENS $_{e} / \mathrm{kW} \cdot h \cdot a^{-1}$ & $S A I D I_{e} / h \cdot a^{-1}$ & $S A I_{e}(\%)$ \\
\hline Scenario 4 & 1276.15 & 5.125 & \\
& & & 99.9415 \\
\hline Scenario 5 & 866.65 & 3.825 & \\
& & & 99.9563 \\
\hline
\end{tabular}

Table 3. System heat reliability indexes in Scenario 1 and Scenario 2

\begin{tabular}{|c|c|c|c|}
\hline Scenario & $E E N S_{h} / k W \cdot h \cdot a^{-1}$ & $S A I D I_{h} / h \cdot a^{-1}$ & SAI $_{h}(\%)$ \\
\hline Scenario 4 & 223.45 & 2.10 & \\
& & & 99.976 \\
\hline Scenario 5 & 212.61 & 2.02 & \\
& & & 99.977 \\
\hline
\end{tabular}

As can be seen from Table 2 and Table 3, when the tie line has interactive power, namely, the fault reduction strategy in Section 2.3 is adopted, and when IES supports the fault load of the distribution network after a distribution network fault is taken into account, compared with Scenario 4, Scenario 5 has a greater improvement in power supply shortage, power load shortage time and power supply reliability indexes. In Scenario 5, the heat supply shortage, heat load shortage time and heating reliability indexes are improved to a certain extent. From the point of view of load shortage, in Scenario 5, compared with Scenario 4, considering the IES and distribution network connection, the absolute value of the expected annual shortage of electric load is reduced by $409.50 \mathrm{~kW} \cdot \mathrm{h}$, a relative decrease of $32.09 \%$; and the absolute value of the expected annual shortage of 
thermal load is reduced by $10.84 \mathrm{~kW} \cdot \mathrm{h}$, a relative decrease of $4.85 \%$. From the perspective of the system energy shortage time, compared with the Scenario 5, the expected absolute value of the annual power supply shortage time of electric load decreases by $1.3 \mathrm{~h}$, which is a relative decrease of $25.37 \%$, and the expected absolute value of the annual power supply shortage time of thermal load decreases by $0.08 \mathrm{~h}$, which is a relative decrease of $3.81 \%$. In these two scenarios, the thermal load only exists inside IES without considering the external thermal network load, and the connection between IES and external network only considers the power system contact. Therefore, the joint optimization scheduling of the system after failure can improve the thermal load reliability to a certain extent, but the improvement effect on the electrical load reliability is more obvious. When the electrical load is at risk of supply shortage, the power interaction between the distribution network and IES can be realized through the tie line. The distribution network dispatches partial load supply and dispatches CHP units, fans, photovoltaic cells and batteries in IES to increase output and reduce the supply shortage of the electrical load. When the thermal load is at risk of supply shortage, part of the electrical load in IES can be supplied interactively through the tieline distribution network and IES electric power, so as to improve the supply continuity of the thermal load by increasing the heating of the electric boiler.

\section{Conclusion}

After the parameters of the component state model are given, the system fault states can be extracted by analytical or simulation methods, and the optimal load reduction modeling based on load classification can be carried out for the distribution network line faults and the IES faults connected to the distribution network respectively, so as to support the fault loads to the maximum extent under the premise of satisfying the economic operation of the system. The sequential Monte Carlo method is used to evaluate the reliability of regional energy Internet, and the reliability evaluation process of the whole system is given. An example is used to verify the effectiveness of the algorithm, and different scenarios are constructed to compare and analyze the load reduction situation under various factors that may affect the reliability index. The results of the example show that the optimal load reduction strategy of regional energy Internet based on load classification proposed in this paper can improve the electrical and thermal reliability indexes of the system, and improve the power supply reliability indexes more greatly.

\section{Acknowledgement}

This work was supported by Technology Project of State Grid Corporation (5204JY190009): Research on Key Technology for Reliability Evaluation of Regional Energy Internet.

\section{References}

1. Wu K, Wang J, Li W, et al (2019). Research on the Operation Mode of New Generation Electric Power System for the Future Energy Internet. Proceedings of the CSEE,39:966-978.

2. Stennikov V, Iakimetc E (2016). Optimal planning of heat supply systems in urban areas. Energy, 110: 157-165.

3. Chen J, Yu T, Xu Y, et al (2019). Fast analytical method for reliability evaluation of electricity-gas integrated energy system considering dispatch strategies. Applied Energy, 242: 260-272.

4. Lei Y, Hou K, Wang Y, et al (2018). A new reliability assessment approach for integrated energy systems: Using hierarchical decoupling optimization framework and impact-increment based state enumeration method. Applied Energy, 210: 12371250 .

5. Su H, Zio E, Zhang J, et al (2020). A systematic method for the analysis of energy supply reliability in complex Integrated Energy Systems considering uncertainties of renewable energies, demands and operations. Journal of Cleaner Production, 267: 122117.

6. Chaudry M, Wu J, Jenkins N(2013). A sequential Monte Carlo model of the combined GB gas and electricity network. Energy Policy, 62: 473-483.

7. Li G, Bie Z, Kou Y, et al (2016). Reliability evaluation of integrated energy systems based on smart agent communication. Applied Energy, 167: 397-406.

8. Talebjedi B, Behbahaninia A (2021). Availability analysis of an Energy Hub with CCHP system for economical design in terms of Energy Hub operator.

9. Chaudry M, Wu J, Jenkins N(2013). A sequential Monte Carlo model of the combined GB gas and electricity network. Energy Policy,62:473-483. 